\title{
A Hemorrhagic Hepatic Cyst Leading to Compression of the Inferior Vena Cava
}

\author{
Yusaku Kajihara* \\ Department of Gastroenterology, Fuyoukai Murakami Hospital, Aomori, Japan
}

A 57-year-old woman with a medical history of alcoholic liver cirrhosis was admitted to the emergency room with epigastric pain and progressive leg edema. Vital signs were normal, and the patient's abdomen was soft. The patient had normal kidney and cardiac function. Esophagogastroduodenoscopy showed neither gastritis nor peptic ulcers. Contrast-enhanced computed tomography (CECT) revealed that a 10.2-cm hepatic cyst had indirectly compressed the inferior vena cava (IVC) (Fig. 1). Six months previously, the cyst size was $8.8 \mathrm{~cm}$, and the IVC was not compressed. Percutaneous transhepatic aspiration of the cyst was performed under sonographic guidance, and $275 \mathrm{~mL}$ of serosanguineous fluid was obtained causing an approximately $2 \mathrm{~cm}$ decrease in the cyst diameter. All symptoms resolved after the procedure, and cytologic examination of the aspirated fluid confirmed no evidence of malignancy. One week later, a follow-up CECT showed improvement of the IVC compression (Fig. 2). Therefore, the intracystic hemorrhage of a simple hepatic cyst leading to a focal narrowing in the IVC was considered the cause of epigastralgia and swelling of the legs. Fortunately, the patient has had no recurrence without any additional treatment for 3 years after the needle aspiration without sclerotherapy.
Although most hepatic cysts are asymptomatic, they can cause serious complications, such as obstructive jaundice, rupture, infection, and intracystic hemorrhages. ${ }^{1}$ Symp-

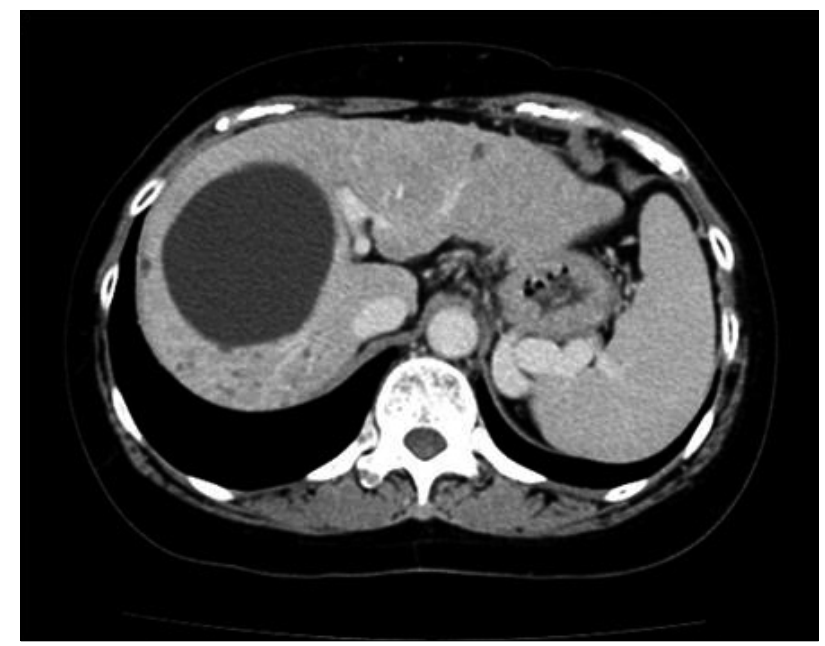

FIG. 2. Follow-up CECT showing improvement of the IVC compression.
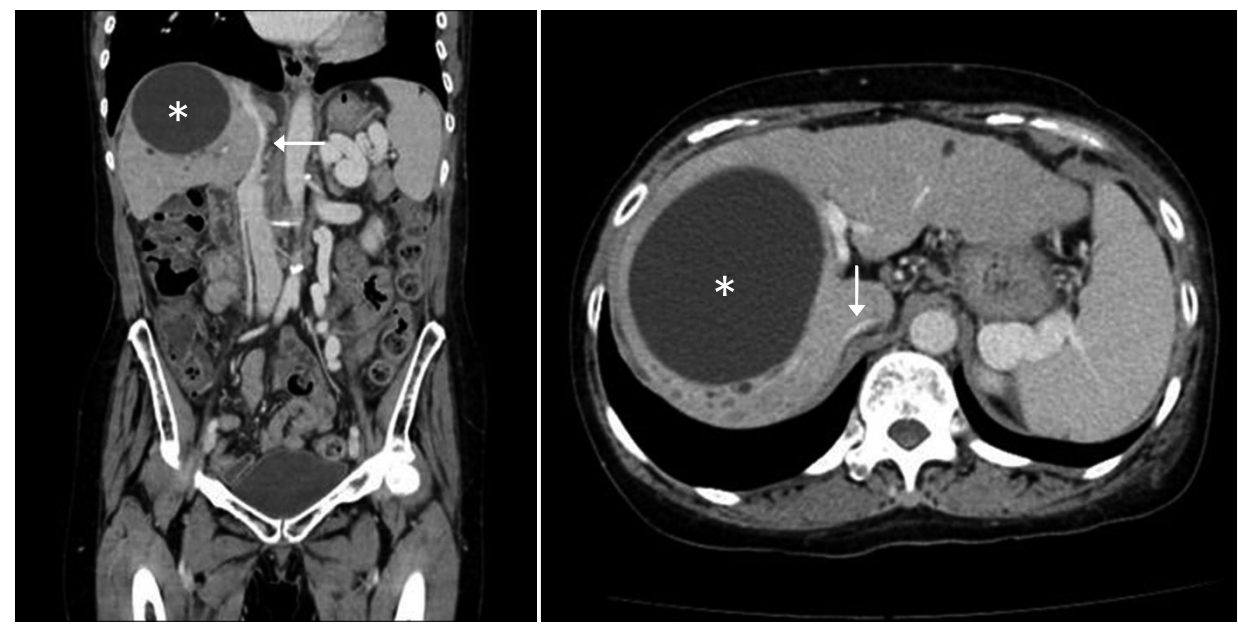

Corresponding Author:

Yusaku Kajihara

Department of Gastroenterology, Fuyoukai Murakami Hospital, 3-3-14 Hamada, Aomori 030-0843, Japan Tel: +81-17-729-8888, Fax: +81-17-729-8887, E-mail: yukajihara-gi@umin.ac.jp
FiG. 1. Contrast-enhanced computed tomography (CECT) revealing that a huge hepatic cyst (white asterisk) had indirectly compressed the inferior vena cava (IVC) (white arrow).
Article History:

Received April 21, 2019

Revised May 6, 2019

Accepted May 7, 2019 
toms of hemorrhagic hepatic cysts include nausea and abdominal pain. ${ }^{2}$ Hepatic cysts may also cause obstructions on the IVC, which may lead to massive edema of the legs. ${ }^{3}$ In most cases, the causes of the intracystic hemorrhage are unclear. ${ }^{2}$ It is important to differentiate hepatobiliary cystic neoplasms from simple hepatic cyst complicated by intracystic hemorrhages. ${ }^{4}$ Treatment options for symptomatic hepatic cysts include surgery and needle aspiration with or without injection of a sclerosing agent into the cyst. ${ }^{3}$

\section{CONFLICT OF INTEREST STATEMENT}

None declared.

\section{REFERENCES}

1. Takahashi G, Yoshida H, Mamada Y, Taniai N, Bando K, Tajiri T. Intracystic hemorrhage of a large simple hepatic cyst. J Nippon Med Sch 2008;75:302-5.

2. Kawano Y, Yoshida H, Mamada Y, Taniai N, Mineta S, Yoshioka $\mathrm{M}$, et al. Intracystic hemorrhage required no treatment from one of multiple hepatic cysts. J Nippon Med Sch 2011;78:312-6.

3. Blonski WC, Campbell MS, Faust T, Metz DC. Successful aspiration and ethanol sclerosis of a large, symptomatic, simple liver cyst: case presentation and review of the literature. World $\mathrm{J}$ Gastroenterol 2006;12:2949-54.

4. Hanazaki K, Wakabayashi M, Mori H, Sodeyama H, Yoshizawa K, Yokoyama S, et al. Hemorrhage into a simple liver cyst: diagnostic implications of a recent case. J Gastroenterol 1997;32:848-51. 